\begin{tabular}{ll} 
Abstract PMO-39 Table 1 & \\
\hline Baseline characteristics & N (\%) \\
\hline Female & $105(54)$ \\
IBD diagnosis & \\
Crohn's Disease & $127(62)$ \\
Ulcerative Colitis & $74(36)$ \\
IBD-unclassified & $3(1)$ \\
Prior biologic & $68(33)$ \\
MDT prior to decision & $57(28)$ \\
Corticosteroid at decision & $63(32)$ \\
Corticosteroid while awaiting initiation & $87(43)$ \\
\hline
\end{tabular}

Conclusions We noted that many patients experience a delay in biologic initiation after a clinical decision to treat. Delay was more likely with a SC route, likely attributable to drug delivery and healthcare at home availability. With the everincreasing demand for biologic therapy in IBD, streamlining the initiation process is imperative to minimise delay in starting effective therapy.

\section{PMO-40 COMPARISON OF THE PARTIAL MAYO AND PUCAI SEVERITY SCORES IN ULCERATIVE COLITIS}

\footnotetext{
1,2Stephanie Shields*, ${ }^{2}$ Konstantinos Gerasimidis, ${ }^{1,2}$ Jonathan Macdonald, ${ }^{3}$ Allan Dunlop, ${ }^{3}$ Peter Galloway, ${ }^{2}$ Vaios Svolos, ${ }^{2} \mathrm{Mr}$ Konstantinos Gkikas, ${ }^{4}$ Richard Hansen, ${ }^{1,2} \mathrm{~J}$ ohn Paul Seenan. 'Department of Gastroenterology, Queen Elizabeth University Hospital, Glasgow, UK; ${ }^{2}$ School of Medicine, Dentistry and Nursing, University of Glasgow, Glasgow, UK; ${ }^{3}$ Department of Biochemistry, Queen Elizabeth University Hospital, Glasgow, UK; ${ }^{4}$ Royal Hospital for Children, Glasgow, UK
}

\subsection{6/gutjinl-2021-BSG.179}

Introduction To assess disease severity in Ulcerative Colitis, the Partial Mayo (P-Mayo) score is used in adults and the Paediatric Ulcerative Colitis Activity Index (PUCAI) score is used for paediatric patients. The P-Mayo score uses an assessment of bowel frequency, rectal bleeding and a physician's global assessment to provide a score. The PUCAI score uses bowel frequency and rectal bleeding, but also incorporates abdominal pain, nocturnal rising, stool consistency and activity levels, arguably providing a more detailed overview. To our knowledge, the scores have never been directly compared. Our aim was to investigate how the scoring systems correlated with each other, and with biomarkers of disease activity.

Methods P-Mayo and PUCAI score, stool and serum samples were collected prospectively from 20 patients at set timepoints between day 0 and 42 after initiation of infliximab therapy. Albumin results were extracted from electronic patient records. Faecal Calprotectin (FC) was measured using a commercially available ELISA assay (CALP0170, Calpro AS). P-Mayo and PUCAI scores were classified as remission, mild, moderate or severe disease activity. R Studio was used to perform Pearson's correlation and agreement was assessed with Cohen's $\kappa$ tests.

Results 134 P-Mayo and 133 PUCAI scores, 131 albumin and 117 FC samples were included from 20 Patients (10 requiring infliximab as rescue therapy having met Travis criteria as inpatients), aged 14-70 (median 38) years. A strong correlation was seen between P-Mayo and PUCAI $(r=0.85, \mathrm{P}<0.0001$, CI 95\%: 0.80, 0.89). Better correlations were seen between PUCAI scores with FC and albumin, when compared to the P-Mayo Score (Table 1). Interclass agreement was moderate

\begin{tabular}{lllll}
\multicolumn{5}{l}{ Abstract PMO-40 Table 1} \\
\hline $\begin{array}{l}\text { Dataset } \\
1\end{array}$ & Dataset & Correlation & $95 \% \mathrm{Cl}$ & P-Value \\
\hline P-Mayo & FC & 0.46 & 0.30, & $<0.0001$ \\
& & & 0.59 & \\
PUCAI & FC & 0.57 & 0.43, & $<0.0001$ \\
& & & 0.68 & \\
P-Mayo & Albumin & -0.35 & $-0.50,-$ & $<0.0001$ \\
& & & 0.19 & \\
PUCAI & Albumin & -0.49 & $-0.62,-$ & $<0.0001$ \\
& & & 0.35 & \\
Mayo & PUCAI & 0.85 & 0.80, & $<0.0001$ \\
& & & 0.89 & \\
\hline
\end{tabular}

between P-Mayo and PUCAI scores $(\mathrm{k}=0.44)$; this improved slightly when mild and moderate disease activity was combined $(\kappa=0.51)$.

Conclusions In this small cohort of mostly adults, P-Mayo and PUCAI correlated strongly with moderate interclass agreement. PUCAI presented stronger correlations than P-Mayo score with blood and colonic disease biomarkers. External validation and evaluation against endoscopy is warranted.

\section{PM0-41 INCORPORATING HLADQA1*05 IN PRE-BIOLOGIC SCREENING IN IBD PATIENTS INITIATING BIOLOGIC THERAPIES}

Haidee Aleman Gonzalez*, Sankaranarayanan Ramachandran, Emma Whitehead, Alison Pattinson, Katie Stamp, Jack Turnbull, Sally Myers, Alison Talbot, Shaji Sebastian. Hull University Teaching Hospitals NHS Trust, Hull, UK

\subsection{6/gutjnl-2021-BSG.180}

Introduction The PANTS study reported high risk of immunogenicity and loss of response in anti Tumor Necrosis Factor (anti-TNFs) treated Crohn's disease (CD) patients carrying HLADQA1*05 allele. The proposed biomarker stratified trial to evaluate the usefulness of HLA testing prior to initiation of anti-TNFs is not yet available.

Aim To evaluate the use of HLADQA1*05 as part of pre-biologic screening in IBD patients initiating biologics on MDT decision on drug choice and disease outcomes

Methods We prospectively included all IBD patients who had HLADQA1*05 tested prior to initiation of biologics over a period of 12 months. Patients with definitive indication for one class of drug or drug strategy (perianal fistula, acute severe colitis, contraindications to infliximab, co-existent EIMs) were excluded. Primary outcome was treatment persistence at 6 and 12 months. Secondary outcomes were steroid free remission at 6 and 12 months, use concomitant immunosuppression and proportion needing dose escalation.

Results Seventy-six patients were included in analysis $(\mathrm{UC}=$ 32, $\mathrm{CD}=43$, IBD-U $=1$ ). HLADQA $1 * 05$ was positive in $46.7 \%$ of patients. The therapy class choice was as recorded in figure 1. Concomitant immunosuppression was used in $44 \%$ of the whole cohort and in $100 \%$ of HLADQA $1 * 05$ positive patients started on anti-TNF agents. Primary nonresponse was recorded in 8 patients and secondary loss of response in 3 patients. Among patients started on anti-TNFs, anti-drug antibodies were detected in 10 (15.6\%) patients 


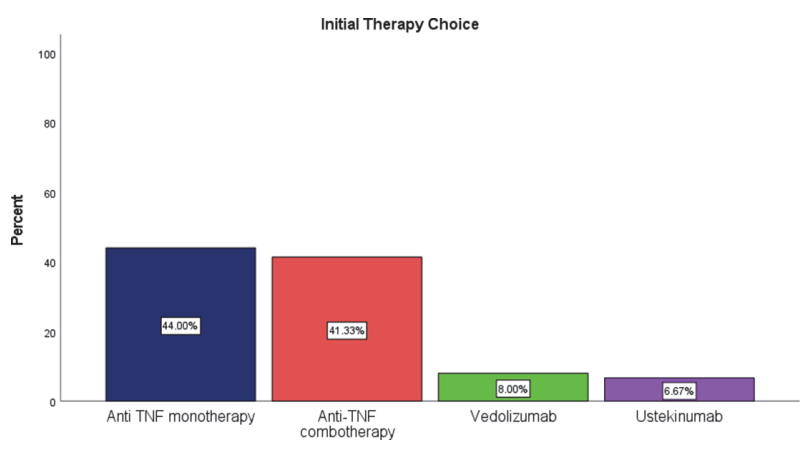

Abstract PM0-41 Figure 1 Initial Theraphy choice

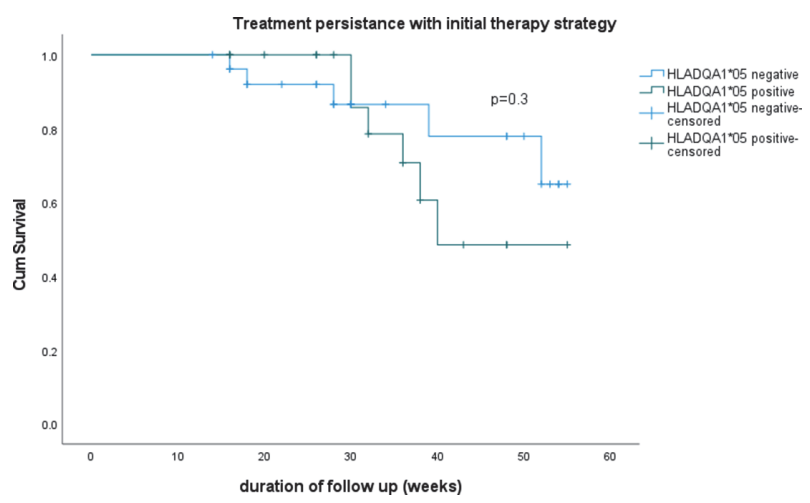

Abstract PM0-41 Figure 2 Treatment presistance with initial theraphy strategy

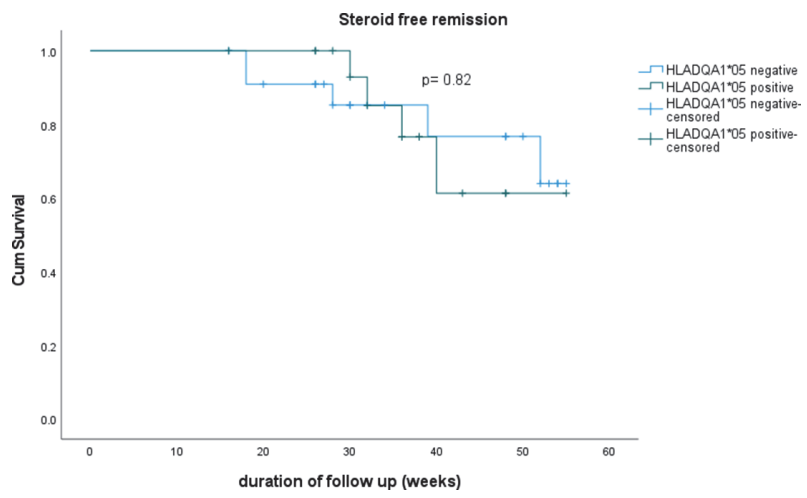

Abstract PM0-41 Figure 3 Steroid free remission

with 7 out of 10 positive for HLADQA $1 * 05$. However, only $3(4.6 \%)$ had undetectable drug levels in the presence of antibody and all three were HLADQA1*05 positive. Two patients had reactions during induction therapy both were HLADQA1*05 positive and were on combination therapy with Infliximab. Therapy persistence with initial drug strategy and steroid free remission at 6 months was recorded in $77.1 \%$ and $78 \%$ respectively. There was no significant difference in drug persistence rates at 6 months and 12 months in patients with HLADQA1*05 variant or those with variant absent (Figure 2). Steroid free remission at 6 and 12 months was also similar irrespective of the variant status (Figure 3)

Conclusions Choice of therapy incorporating HLADQA1*05 status may allow anti-TNF monotherapy and tailoring of therapy in IBD patients. A randomised stratified biomarker trial is required to determine the utility of pre-treatment testing.
PM0-42 ACCELERATED DOSING SCHEDULE WITH USTEKINUMAB IN ANTI-TNF REFRACTORY CROHN'S DISEASE

Haidee Aleman Gonzalez*, Katie Stamp, Emma Whitehead, Sally Myers, Jack Turnbull, Alison Pattinson, Alison Talbot, Shaji Sebastian. Hull University Teaching Hospitals Nhs Trust, Hull, UK

\subsection{6/gutjnl-2021-BSG.181}

Introduction The efficacy of ustekinumab in prior biologic exposed refractory patients with inflammatory bowel disease is significantly inferior to those who are bio naïve. The optimal therapeutic level and hence the optimal dosing strategy in this setting is uncertain. Whether early dose optimisation is beneficial in this group of patients has not been evaluated. We aimed to compare the effectiveness and safety of early dose optimisation for maintenance compared to conventional maintenance regime in a retrospective cohort of refractory IBD patients.

Methods All patients initiated on ustekinumab following failure of one or more anti-TNF agents were included. Patients who received conventional maintenance regime (Q8W) was compared to those who received accelerated maintenance regime $(\mathrm{Q} 4 \mathrm{H})$. The primary end point was steroid free remission at 12 months or at last follow up. The secondary end points were ustekinumab persistence at 6 months and 12 months, need for surgery and drug related infectious or non-infectious complications.

Results One hundred and four patients were included (male: female 48:56). Median follow up was 11 months (IQR 5-14). Fifty-six patients received accelerated dosing regimen while 48 patients received standard dosing regime. Dose escalation was required in 18 patients $(37.5 \%)$ of standard dosing regimen while four patients $(7.1 \%)$ in the accelerated dosing regimen had dose de-escalation. Higher proportion patients in the accelerated dosing regimen were in steroid free remission $87.5 \%$ vs $68.8 \%(p=0.018)$. Proportion of patients requiring surgery was higher in the standard dosing regimen group (22.91 5 vs $7.14 \%, p=0.02)$. Ustekinumab persistence in patient started on the accelerated regime and conventional dosing regime was not significantly different at 6 months $(94.1 \%$ and $95.3 \%, p=0.58)$ and 12 months $(84.4 \%$ and 95.3, $\mathrm{p}=0.1)$. No serious drug related complications observed in either group.

Conclusions Early dose optimisation of Ustekinumab improves steroid free remission rates and reduces the need for surgery in patients with anti-TNF refractory patients with IBD.

\section{PMO-43 COLIFORM-SENSITIVITY BASED ORAL ERTAPENEM THERAPY FOR CHRONIC ANTIBIOTIC-REFRACTORY POUCHITIS: AN UNDERUTILISED OPTION?}

Thomas Conley*, Ibrahim Mian, Kate Martin, Paul Rooney, Sreedhar Subramanian. Liverpool University Foundation Trust, Liverpool, UK

\subsection{6/gutjnl-2021-BSG.182}

Introduction Up to $50 \%$ of patients have at least one episode of pouchitis after ileal pouch anal-anastomosis (IPAA). Among these, a proportion develop chronic antibiotic-dependent or chronic antibiotic refractory pouchitis (CARP). Pouch failure is common among patients with CARP. Faecal coliform sensitivity testing in such patients can identify a subgroup in whom an alternative, more appropriate antimicrobial agent can be utilised. We previously reported successful coliform sensitivity- 